\title{
A nonlinear Luenberger-like observer for nonlinear singular systems
}

\author{
Gang Zheng ${ }^{\mathrm{a}, \mathrm{b}, \mathrm{c}}$, Driss Boutat ${ }^{\mathrm{d}}$, Haoping Wang ${ }^{\mathrm{a}}$ \\ ${ }^{a}$ International Sino-French Laboratory of Automatic Control and Signal Processing(LaFCAS), \\ School of Automation, Nanjing University of Science and Technology, China \\ ${ }^{b}$ INRIA - Lille Nord Europe, 40 avenue Halley, Villeneuve d'Ascq 59650, France. \\ ${ }^{c}$ CRIStAL, CNRS UMR 9189, Ecole Centrale de Lille, BP 48, 59651 Villeneuve d'Ascq, France. \\ ${ }^{d}$ Loire Valley University, ENSI de Bourges, Laboratoire PRISME, 88, Boulevard de Lahitolle, 18020, France.
}

\begin{abstract}
This paper investigates observer design problem for a large class of nonlinear singular systems with multiple outputs. We firstly regularize the singular system by injecting the derivative of outputs into the system. Then differential geometric method is applied to transform the regularized system into a simple normal form, for which a Luenberger-like observer is proposed.
\end{abstract}

\section{Introduction}

Singular systems widely exist in engineering systems, such as chemical system, biological system, electrical circuit and so on. These systems are governed by mixing differential and algebraic equations, which are the special difference with respect to regular systems, thus the control of such system is a challenging problem (see Dai (1989), Campbell (1982), Campbell (1980)). Due to this reason, many well-defined concepts relative to observation problem for regular (non-singular) systems have to be reconsidered for singular ones.

The solvability, controllability and observability concepts have been studied in Yip and Sincovec (1981) for singular systems with regular matrix pencil. Causal observability has been treated in Hou and Muller (1999a) for linear singular systems. Recently, Bejarano et al. (2013) generalizes the observability for linear singular system to consider the unknown input case, by converting the singular system into a regular one with unknown inputs and algebraic constraints. Moreover, the assumption of regular matrix pencil for singular systems was removed as well in Bejarano et al. (2011, 2013). It has been extended in Bejarano et al. (2012) and Bejarano et al. (2015) to treat nonlinear singular systems.

Concerning the observer design, a Luenberger-like observer has been proposed in Paraskevopoulos and Koumboulis (1992) for linear singular systems. Darouach and Boutayeb (1995) gave necessary and sufficient condition for the existence of a reduced-order observer for linear singular systems with known inputs. In Hou and Muller (1999b), a generalized observer was studied by involving the derivative of input and output. For

\footnotetext{
This paper was supported in part by Ministry of Higher Education and Research Nord-Pas de Calais Regional Council, by FEDER through the Contrat de Projets Etat Region (CPER), France, International associated Laboratory of LaFCAS, International Science and Technology Cooperation Program of China project (2015DFA01710) and Jiangsu Science Foundation Fund of China, BK20170094.

E-mail address: hp.wang@njust.edu.cn (H.Wang).
}

linear singular systems with unknown input, a proportionalintegral observer was proposed in Koenig and Mammar (2002), and its extension by involving multiple integrations to design unknown input observer for linear singular systems with unknown input was studied in Koenig (2005) and Gao and Ho (2004). For nonlinear singular systems, Kaprielian and Turi (1992) studied an observer in which the system was linearized around the equilibrium point. The same technique was used in Boutayeb and Darouach (1995) to study the reduced-order observer for a class of nonlinear singular systems. Also, many efforts have been made when the system is affected by some disturbances in input, in the model, or in the measurement. In Gao and Ho (2006), a simple linear singular observer was proposed, and necessary and sufficient conditions were given for a special class of linear singular systems with unknown inputs. This result was extended as well to treat nonlinear singular systems under the assumption that the nonlinear term is Lipschitz. Other techniques, such as LMI Lu and Ho (2006), Darouach and Boutat-Baddas (2008), Lu et al. (2004) and convex optimization Koenig (2006), are proposed as well to design observer for nonlinear singular systems with known (or unknown) inputs. Recently, the technique of regularization by applying the differential geometric method (Zheng et al. (2007, 2009); Zheng and Boutat (2011); Tami et al. (2013, 2016)) to nonlinear singular systems was introduced in Boutat et al. (2012). But this method works only for nonlinear singular systems with single output. This paper is an extension of that result to treat multiple outputs case. Given a nonlinear singular system, we firstly regularize it into a nonlinear regular system with the injection of the output derivative, then seek a diffeomorphism to transform the regularized system into an observer normal form, based on which a Luenberger-like observer is proposed. Compared to the existing results on nonlinear singular systems whose nonlinear terms need to be Lipschitz Shields (1997); Gao and Ho (2006); Darouach and Boutat-Baddas (2008), the presented technique works as well when this assumption is not satisfied.

This paper is organized as follows. Section 2 recalls basic 
results of Luenberger-like observer for linear singular systems. Section 3 presents the method how to regularize nonlinear singular system into a regular one with output derivatives. Section 4 deduces necessary and sufficient conditions to transform the regularized systems into a simple normal form, for which a Luenberger-like observer has been proposed in Section 5.

\section{Recall for linear singular system}

Firstly, let us recall some basic results for linear singular systems. Consider a general linear singular system as follows:

$$
\left\{\begin{array}{l}
\bar{E} \dot{\zeta}=\bar{A} \zeta \\
\bar{y}=\bar{C} \zeta
\end{array}\right.
$$

with $\zeta \in \mathbb{R}^{n}, \operatorname{rank} \bar{E}<n$, and it is assumed that the matrix pencil $s \bar{E}-\bar{A}$ is regular. In Darouach and Boutayeb (1995), the following two conditions are proposed:

$$
\operatorname{rank}\left[\begin{array}{cc}
\bar{E} & \bar{A} \\
0 & \bar{E} \\
0 & \bar{C}
\end{array}\right]=n+\operatorname{rank} \bar{E}
$$

and

$$
\operatorname{rank}\left[\begin{array}{c}
s \bar{E}-\bar{A} \\
\bar{C}
\end{array}\right]=n, \forall s \in \mathbb{C}, \operatorname{Re}(s) \geq 0
$$

which guarantee the existence of a simple Luenberger-like observer

$$
\left\{\begin{array}{l}
\dot{\xi}=N \xi+L y \\
\hat{\zeta}=\xi+K y
\end{array}\right.
$$

with properly chosen matrices $N, K$ and $L$.

The following section will show how to generalize this idea to treat nonlinear singular systems. Similar conditions as those for linear singular systems will be proposed, and we will show that those conditions coincide with the above two conditions (2) and (3) if the linear case is studied.

\section{Regularization of nonlinear singular systems}

Consider the following class of nonlinear singular systems:

$$
\left\{\begin{array}{l}
\bar{E} \dot{\zeta}=\bar{f}(\zeta) \\
\bar{y}=\bar{h}(\zeta)
\end{array}\right.
$$

where $\zeta \in \Omega_{\zeta} \in \mathbb{R}^{n}, \bar{f}: \mathbb{R}^{n} \rightarrow \mathbb{R}^{n}$ and $\bar{h}=\left[\bar{h}_{1}, \cdots, \bar{h}_{m}\right]^{T}$ : $\mathbb{R}^{n} \rightarrow \mathbb{R}^{m}$ are smooth, with $\bar{E} \in \mathbb{R}^{n \times n}$ being singular, i.e. $\operatorname{rank} \bar{E}<n$. Without loss of generalities, it is assumed that $\left\{d \bar{h}_{1}(\zeta), \cdots, d \bar{h}_{m}(\zeta)\right\}$ are linearly independent for all $\zeta \in \Omega_{\zeta}$ where $d \bar{h}_{1}$ means the differential of $\bar{h}_{1}$. It is worth noting that a corresponding regularity assumption is not required for the studied nonlinear system (5).

Assumption 1. For any $\zeta \in \Omega_{\zeta}$, it is assumed that the following condition

$$
\operatorname{rank}\left[\begin{array}{cc}
\bar{E} & \frac{\partial \bar{f}(\zeta)}{\partial \zeta} \\
0 & \bar{E} \\
0 & \frac{\partial \bar{h}(\zeta)}{\partial \zeta}
\end{array}\right]=n+\operatorname{rank} \bar{E}
$$

is satisfied.
Remark 1. The equation (6) in Assumption 1 can be seen as a generalization of (2) to treat nonlinear singular system. In fact, if we consider the linear case of (5), i.e. $\bar{f}(\zeta)=\bar{A} \zeta$ and $\bar{h}(\zeta)=\bar{C} \zeta$, the equation (6) becomes exactly (2):

$$
\operatorname{rank}\left[\begin{array}{cc}
\bar{E} & \frac{\partial \bar{f}(\zeta)}{\partial \zeta} \\
0 & \bar{E} \\
0 & \frac{\partial \bar{h}(\zeta)}{\partial \zeta}
\end{array}\right]=\operatorname{rank}\left[\begin{array}{cc}
\bar{E} & \bar{A} \\
0 & \bar{E} \\
0 & \bar{C}
\end{array}\right]=n+\operatorname{rank} \bar{E}
$$

which is the necessary and sufficient condition of observability for linear singular systems.

Note $\operatorname{rank} \bar{E}=q<n$, then there exist two elementary matrices $T$ and $S$ such that

$$
S \bar{E} T=\left[\begin{array}{cc}
I_{q} & 0 \\
0 & 0
\end{array}\right]
$$

thus, by introducing $x=T^{-1} \zeta: \Omega_{\zeta} \rightarrow \Omega_{x}$, system (5) becomes

$$
\left\{\begin{array}{l}
S \bar{E} T \dot{x}=S \bar{f}(T x) \\
\bar{y}=\bar{h}(T x)
\end{array}\right.
$$

which is equivalent to

$$
\left\{\begin{array}{l}
E \dot{x}=\tilde{f}(x) \\
\bar{y}=\tilde{h}(x)
\end{array}\right.
$$

with $E=\left[\begin{array}{cc}I_{q} & 0 \\ 0 & 0\end{array}\right], \tilde{f}(x)=S \bar{f}(T x)$ and $\tilde{h}(x)=\bar{h}(T x)$, or is equivalent to the following decomposition:

$$
\left\{\begin{array}{l}
\dot{x}_{1}=\tilde{f}_{1}\left(x_{1}, x_{2}\right) \\
0=\tilde{f}_{2}\left(x_{1}, x_{2}\right) \\
\bar{y}=\tilde{h}\left(x_{1}, x_{2}\right)
\end{array}\right.
$$

where $x=\left[\begin{array}{c}x_{1} \\ x_{2}\end{array}\right], x_{1} \in \mathbb{R}^{q}, x_{2} \in \mathbb{R}^{n-q}, \tilde{f}=\left[\begin{array}{c}\tilde{f}_{1} \\ \tilde{f}_{2}\end{array}\right]$.

Remark 2. For system (9), if

$$
\operatorname{rank} \frac{\partial \tilde{f}_{2}(x)}{\partial x_{2}}=n-q, \forall x \in \Omega_{x}
$$

then implicit function theorem ensures that there exists a function $\alpha$ such that $x_{2}=\alpha\left(x_{1}\right)$. By inserting it back into (9), the following reduced regular model can be obtained

$$
\left\{\begin{array}{l}
\dot{x}_{1}=\tilde{f}_{1}\left(x_{1}, \alpha\left(x_{1}\right)\right) \\
\bar{y}=\tilde{h}\left(x_{1}, \alpha\left(x_{1}\right)\right)
\end{array}\right.
$$

Then the classical differential geometric method can be used to analyze observability and design observer. However, condition (10) is in some sense a little strong since it requires that the algebraic constrain contains all information of $x_{2}$. Otherwise, the reduced-order regularized system cannot be obtained. From (9), it can be seen clearly that $\bar{y}$ contains as well the information of $x_{2}$. Therefore, one natural way to relax condition (10) is to take into account as well the output of (9). 
Lemma 1. If Assumption 1 is satisfied, then the following equation

$$
\operatorname{rank}\left[\begin{array}{c}
\frac{\partial \tilde{f}_{2}(x)}{\partial x_{2}} \\
\frac{\partial \tilde{h}(x)}{\partial x_{2}}
\end{array}\right]=n-q, \forall x \in \Omega_{x}
$$

is satisfied.

Proof. Since the proof of this lemma is straightforward, thus it is omitted.

Moreover, if $\operatorname{rank}\left[\begin{array}{c}\frac{\partial \tilde{f}_{2}(x)}{\partial x_{2}} \\ \frac{\partial \tilde{h}(x)}{\partial x_{2}}\end{array}\right]=n-q$, then there exists an invertible matrix $Q(x)=\left[\begin{array}{l}Q_{1}(x) \\ Q_{2}(x)\end{array}\right]$ with dimension $(m+n-q) \times(m+n-q)$ for all $x \in \Omega_{x}$ such that

$$
Q(x)\left[\begin{array}{c}
\frac{\partial \tilde{f}_{2}(x)}{\partial x_{2}} \\
\frac{\partial \tilde{h}(x)}{\partial x_{2}}
\end{array}\right]=\left[\begin{array}{c}
I_{n-q} \\
0_{m}
\end{array}\right]
$$

with $Q_{1}(x)\left[\begin{array}{c}\frac{\partial \tilde{f}_{2}(x)}{\partial x_{2}} \\ \frac{\partial \tilde{h}(x)}{\partial x_{2}}\end{array}\right]=I_{n-q}$. From the algebraic equation in (9), its derivative with respect to time yields

$$
\left[\begin{array}{c}
0 \\
\dot{\bar{y}}
\end{array}\right]=\left[\begin{array}{c}
\frac{\partial \tilde{f}_{2}(x)}{\partial x} \\
\frac{\partial \tilde{h}(x)}{\partial x}
\end{array}\right] \dot{x}=\left[\begin{array}{c}
\frac{\partial \tilde{f}_{2}(x)}{\partial x_{1}} \\
\frac{\partial \tilde{h}(x)}{\partial x_{1}}
\end{array}\right] \tilde{f}_{1}(x)+\left[\begin{array}{c}
\frac{\partial \tilde{f}_{2}(x)}{\partial x_{2}} \\
\frac{\partial \tilde{h}(x)}{\partial x_{2}}
\end{array}\right] \dot{x}_{2}
$$

which gives

$$
\dot{x}_{2}=-Q_{1}(x)\left[\begin{array}{c}
\frac{\partial \tilde{f}_{2}(x)}{\partial x_{1}} \\
\frac{\partial \tilde{h}(x)}{\partial x_{1}}
\end{array}\right] \tilde{f}_{1}(x)+Q_{1}(x)\left[\begin{array}{c}
0 \\
\dot{\bar{y}}
\end{array}\right]
$$

By using this artificial dynamics of $\dot{x}_{2}$, system (9) can be regularized as

$$
\left\{\begin{array}{l}
\dot{x}=f(x)+g(x) \dot{y} \\
y=h(x)
\end{array}\right.
$$

with

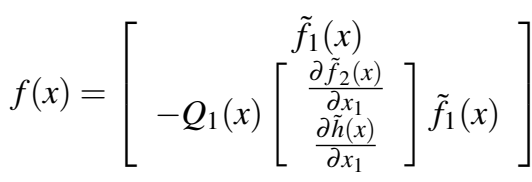

$$
\begin{aligned}
& g(x)=\left[\begin{array}{c}
0 \\
Q_{1}(x)
\end{array}\right]
\end{aligned}
$$

and

$$
y=\left[\begin{array}{l}
0 \\
\bar{y}
\end{array}\right], h(x)=\left[\begin{array}{c}
\tilde{f}_{2}(x) \\
\tilde{h}(x)
\end{array}\right]
$$

It is worth noting that the new output $y$ is the combination of the original one $\bar{y}$ and the algebraic equation hidden in (5), and this idea has been firstly proposed in Darouach and Boutayeb (1995).

For the sake of simplicity, it is assumed for the regularized form (13) that the first $\bar{m}$ outputs are independent with $\bar{m} \leq n+m-q$, i.e., $\left\{d h_{1}(x), \cdots, d h_{\bar{m}}(x)\right\}$ are linearly independent for all $x \in \Omega_{x}$. If it is not the case, then some simple manipulations enable us to remove the dependent outputs and put them in order. We can then impose the following assumption.
Assumption 2. It is assumed that the regularized system (13) is locally observable in the sense that there exist $r_{i}$ for $1 \leq i \leq \bar{m}$ such that $\sum_{i=1}^{\bar{m}} r_{i}=n$ and

$$
\operatorname{rank}\left[\begin{array}{c}
d h_{1}(x) \\
\vdots \\
d L_{f(x)}^{r_{i}-1} h_{1}(x) \\
\vdots \\
d h_{\bar{m}}(x) \\
\vdots \\
d L_{f(x)}^{r_{\bar{m}}-1} h_{\bar{m}}(x)
\end{array}\right]=n, \forall x \in \Omega_{x}
$$

where $L_{f(x)}^{k} h_{i}(x)$ for $1 \leq k \leq r_{i}$ represents the classical Lie derivative.

The following proposition shows that the above assumption is a natural generalization of condition (3) for linear singular systems.

Proposition 1. For system (5), if $\bar{f}(\xi)=\bar{A} \xi$ and $\bar{h}(\xi)=\bar{C} \xi$, then condition (17) implies condition (3) if Assumption 1 is satisfied.

Proof. By setting $\bar{f}(\xi)=\bar{A} \xi$ and $\bar{h}(\xi)=\bar{C} \xi$, it is clear that there exist two elementary matrices $T$ and $S$, with the change of coordinates $x=T \zeta$, system (5) can be transformed into

$$
\left\{\begin{array}{l}
E \dot{x}=\tilde{A} x \\
\bar{y}=\tilde{C} x
\end{array}\right.
$$

with $E=\left[\begin{array}{cc}I_{q} & 0 \\ 0 & 0\end{array}\right], \tilde{A}=S \bar{A} T^{-1}$ and $\tilde{C}=\bar{C} T^{-1}$. Note $E_{q}=$ $\left[I_{q}, 0\right], \tilde{A}=\left[\begin{array}{c}\tilde{A}_{1} \\ \tilde{A}_{2}\end{array}\right]$ and $C=\left[\begin{array}{c}\tilde{A}_{2} \\ \tilde{C}\end{array}\right]$, then system (18) can be decomposed as

$$
\left\{\begin{array}{l}
E_{q} \dot{x}=\dot{x}_{1}=\tilde{A}_{1} x \\
y=C x
\end{array}\right.
$$

with $x_{1} \in \mathbb{R}^{q}$ and $y=\left[\begin{array}{l}0 \\ \bar{y}\end{array}\right]$.

Decompose the matrix $C$ as $C=\left[C_{1}, C_{2}\right]$. Therefore, if Assumption 1 is satisfied, then according to Lemma 1 we have rank $C_{2}=n-q$ which is full row rank, thus there exists an invertible matrix $Q_{1}$ such that $Q_{1} C_{2}=I_{n-q}$.

Also, we have $\operatorname{rank}\left[\begin{array}{c}E_{q} \\ C\end{array}\right]=\operatorname{rank}\left[\begin{array}{cc}I_{q} & 0 \\ C_{1} & C_{2}\end{array}\right]=n$, which implies the existence of an invertible matrix $P=$ $\left[\begin{array}{cc}I_{q} & 0 \\ -Q_{1} C_{1} & Q_{1}\end{array}\right]$ such that $P\left[\begin{array}{c}E_{q} \\ C\end{array}\right]=I_{n}$.

Rewriting system (19) as

$$
\left\{\begin{array}{l}
E_{q} \dot{x}=\tilde{A}_{1} x \\
C \dot{x}=\dot{y}
\end{array}\right.
$$

which leads, by multiplying both side the invertible matrix $P$, to the following output derivative form

$$
\left\{\begin{array}{l}
\dot{x}=A x+\left[\begin{array}{l}
0 \\
\dot{y}
\end{array}\right] \\
y=C x
\end{array}\right.
$$


with $A=\left[\begin{array}{c}\tilde{A}_{1} \\ -Q_{1} C_{1} \tilde{A}_{1}\end{array}\right]$. Thus, if we take $\bar{f}(\xi)=\bar{A} \xi$ and $\bar{h}(\xi)=\bar{C} \xi$ in system (5), then condition (17) is equivalent to state that the pair $(A, C)$ is observable, or equivalently

$$
\operatorname{rank}\left[\begin{array}{c}
s I_{n}-A \\
C
\end{array}\right]=n, \forall s \in \mathbb{C}
$$

Following the proof of Theorem 2.1 in Darouach and Boutayeb (1995), we have

$$
\begin{aligned}
& \operatorname{rank}\left[\begin{array}{c}
s I_{n}-A \\
C
\end{array}\right]=\operatorname{rank}\left[\begin{array}{c}
s E_{q}-\tilde{A}_{1} \\
s\left[0, I_{n-q}\right]+Q_{1} C_{1} \tilde{A}_{1} \\
C
\end{array}\right] \\
& =\operatorname{rank}\left[\begin{array}{c}
s E_{q}-\tilde{A}_{1} \\
s\left[0, Q_{1} C_{2}\right]+Q_{1} C_{1} \tilde{A}_{1} \\
C
\end{array}\right] \\
& =\operatorname{rank}\left[\begin{array}{c}
s E_{q}-\tilde{A}_{1} \\
s Q_{1} C-s Q_{1} C_{1} E+Q_{1} C_{1} \tilde{A}_{1} \\
C
\end{array}\right] \\
& =\operatorname{rank}\left[\begin{array}{ccc}
I_{q} & 0 & 0 \\
-Q_{1} C_{1} & Q_{1} & 0 \\
0 & 0 & I_{n-q+m}
\end{array}\right]\left[\begin{array}{c}
s E_{q}-\tilde{A}_{1} \\
s C \\
C
\end{array}\right] \\
& =\operatorname{rank}\left[\begin{array}{c}
s E_{q}-\tilde{A}_{1} \\
C
\end{array}\right]=\operatorname{rank}\left[\begin{array}{c}
s E_{q}-\tilde{A}_{1} \\
-\tilde{A}_{2} \\
\tilde{C}
\end{array}\right]=\operatorname{rank}\left[\begin{array}{c}
s E-\tilde{A} \\
\tilde{C}
\end{array}\right] \\
& =\operatorname{rank}\left[\begin{array}{cc}
S & 0 \\
0 & I_{m}
\end{array}\right]\left[\begin{array}{c}
s \bar{E}-\bar{A} \\
\bar{C}
\end{array}\right]\left[\begin{array}{cc}
T^{-1} & 0 \\
0 & I_{m}
\end{array}\right] \\
& =\operatorname{rank}\left[\begin{array}{c}
s \bar{E}-\bar{A} \\
\bar{C}
\end{array}\right]=n
\end{aligned}
$$

for all $s \in \mathbb{C}$. Finally we proved that, if $\bar{f}(\xi)=\bar{A} \xi$ and $\bar{h}(\xi)=$ $\bar{C} \xi$ for system (5), then condition (17) implies condition (3) if Assumption 1 is satisfied.

Remark 3. Let us remark that, even for linear singular systems, condition (17) only implies condition (3), but not equivalent, since the deduced condition from (17) imposes observability for all states, i.e. for all $s \in \mathbb{C}$, but condition (3) asks only detectability, i.e. for all $s \in \mathbb{C}, \operatorname{Re}(s) \geq 0$. This gap can be fixed by only requiring that the internal dynamics of (13) is stable Isidori (1995). In such a way, condition (17) is exactly equivalent to (3) for linear case. For the sake of simplicity, this paper does not treat this issue, but the same idea can be easily generalized to treat this case.

\section{Observer normal form}

Supposed that Assumptions 1 and 2 are both satisfied, then system (5) can be put into

$$
\left\{\begin{array}{l}
\dot{x}=f(x)+g(x) \dot{y} \\
y=h(x)
\end{array}\right.
$$

where $f, g$ and $h$ are respectively defined in (14), (15) and (16), with $\left\{d h_{1}(x), \cdots, d h_{\bar{m}}(x)\right\}$ are linearly independent for all $x \in$ $\Omega_{x}$.
Remark 4. For the regularized system (20), different techniques can be used to design observers by imposing different kinds of conditions, such as Lyapunov Boutayeb and Darouach (1995), LMI Shields (1997); Gao and Ho (2006); Darouach and Boutat-Baddas (2008), convex optimization Koenig (2006) and so on. Almost all existing results on observer design for nonlinear singular systems, Lipschitz condition for the contained nonlinear term is always imposed to facilitate the design of observers. However, this paper focuses on applying conventional differential geometric method to design observer. This technique enables us to avoid the Lipschitz assumption, but of course other assumptions will be imposed. More precisely, we will firstly seek a diffeomorphism $z=\phi(x)$ to transform the regularized system (20) into an observer normal form, and then design a Luenberger-like observer for this observer normal form.

Following the conventional procedure of differential geometric method, let us note $\theta_{i, k}=d L_{f(x)}^{k-1} h_{i}(x)$ for $1 \leq i \leq \bar{m}$ and $1 \leq k \leq r_{i}$, and

$$
\Delta=\operatorname{span}\left\{\theta_{j, k}, 1 \leq j \leq \bar{m} \text { and } 1 \leq k \leq r_{j}\right\}
$$

then we can define the following family of vector fields $\left(\tau_{i, 1}\right)_{1 \leq i \leq \bar{m}}$ such that:

$$
\begin{aligned}
& \theta_{i, r_{i}}\left(\tau_{i, 1}\right)=1 \text { for } 1 \leq i \leq \bar{m} \\
& \theta_{i, k}\left(\tau_{i, 1}\right)=0 \text { for } 1 \leq k \leq r_{i}-1 \\
& \theta_{j, k}\left(\tau_{i, 1}\right)=0 \text { for } j<i \text { and } 1 \leq k \leq r_{j} \\
& \theta_{j, k}\left(\tau_{i, 1}\right)=0 \text { for } j>i \text { and } 1 \leq k \leq r_{j}
\end{aligned}
$$

Since it is assumed that (17) is satisfied, then each family of vector fields $\left(\tau_{i, 1}\right)_{1 \leq i \leq \bar{m}}$ defined by the above equation (22) can yield the frame $\tau=\left(\cdots, \tau_{i, j}, \cdots\right)_{1 \leq i \leq \bar{m}, 1 \leq j \leq r_{i}}$, where

$$
\tau_{i, k}=\left[\tau_{i, k-1}, f(x)\right] \text { for } 1 \leq i \leq \bar{m} \text { and } 2 \leq k \leq r_{i} .
$$

Denote

$$
\theta=\left(\theta_{1,1}, \cdots, \theta_{1, r_{1}}, \cdots, \theta_{\bar{m}, 1}, \cdots, \theta_{\bar{m}, r_{\bar{m}}}\right)^{T}
$$

and set $\Lambda=\theta(\tau)$. Due to the observability rank condition (17), the matrix $\Lambda$ is invertible, hence one can define the following multi 1 -forms

$$
\omega=\Lambda^{-1} \theta
$$

Then the following result is a generalization of Theorem 3.4 in Xiao and Gao (1989).

Theorem 1. Supposed that Assumptions 1 and 2 are satisfied. Then there exists a local diffeomorphism $z=\phi(x)$ for $x \in \bar{\Omega}_{x} \subseteq \Omega_{x}$ which transforms system (13) into the following output-derivative-injection normal form:

$$
\left\{\begin{array}{l}
\dot{z}_{i}=A_{i} z_{i}+\alpha_{i}(y)+\beta_{i}(y) \dot{y}, \text { for } 1 \leq i \leq \bar{m} \\
y_{i}=C_{i} z_{i}=z_{i, r_{i}}
\end{array}\right.
$$

with $z=\left[z_{1}^{T}, \cdots, z_{\bar{m}}^{T}\right]^{T}, A_{i}$ being Brounovsky form

$$
A_{i}=\left(\begin{array}{ccccc}
0 & \cdots & 0 & 0 & 0 \\
1 & \cdots & 0 & 0 & 0 \\
\vdots & \ddots & \ddots & \ldots & \vdots \\
0 & \cdots & 1 & 0 & 0 \\
0 & \cdots & 0 & 1 & 0
\end{array}\right)
$$


for $1 \leq i \leq \bar{m}, \sum_{i=1}^{\bar{m}} r_{i}=n, \alpha_{i}$ and $\beta_{i}$ being respectively $r_{i}$ and $r_{i} \times \bar{m}$ matrix, if and only if

- $\left[\tau_{i, j}, \tau_{s, l}\right]=0$, for $1 \leq i \leq \bar{m}, 1 \leq j \leq r_{i}, 1 \leq s \leq \bar{m}$ and $1 \leq l \leq r_{s}$

- $\operatorname{dim}\left[\Delta_{i}\right]=\operatorname{dim}\left[\Delta_{i} \cap \Delta\right]$ for $1 \leq i \leq \bar{m}$, where $\Delta$ is defined in (21) and

$$
\begin{aligned}
\Delta_{i}= & \operatorname{span}\left\{d L_{f}^{k-1} h_{j} ; 1 \leq j \leq \bar{m},\right. \\
& \left.1 \leq k \leq r_{i}, \quad d L_{f}^{r_{i}-1} h_{i} \text { is omitted }\right\}
\end{aligned}
$$

- $\left[\tau_{i, j}, g_{k}(x)\right]=0$ for $1 \leq i \leq \bar{m}, 1 \leq j \leq r_{i}-1,1 \leq k \leq \bar{m}$.

Proof. In Xiao and Gao (1989), it has been proven that the first two conditions in Theorem 1 are necessary and sufficient to guarantee the existence of a local diffeomorphism $z=\phi(x)$ for $x \in \bar{\Omega}_{x} \subseteq \Omega_{x}$ such that (20) without the term $g(x) \dot{y}$ can be transformed into

$$
\left\{\begin{array}{l}
\dot{z}_{i}=A_{i} z_{i}+\alpha_{i}(y), \text { for } 1 \leq i \leq \bar{m} \\
y_{i}=C_{i} z_{i}=z_{i, r_{i}}
\end{array}\right.
$$

The rest needs only to prove that the third item of Theorem 1 is the necessary and sufficient condition to transform $g_{k}(x)$ into $\beta_{k}(y)$ for $1 \leq k \leq \bar{m}$.

Necessity: It is assumed that there exists a local diffeomorphism $z=\phi(x)$ for $x \in \bar{\Omega}_{x} \subseteq \Omega_{x}$ which transforms (13) into (25), then the following will prove that system (13) should satisfy the third item of Theorem 1.

To this aim, note $\omega=d \phi$, thus $\omega_{i}$ for $1 \leq i \leq \bar{m}$ are the pullbacks of $d z_{i}$, i.e. ${ }^{1} \omega_{i}=\phi^{*}(d \xi)=d \phi_{i}$.

In the following, we will firstly check that the third item of Theorem 1 is satisfied for the transformed normal form (25), then we will use the property of pullback to prove the necessity for the original system (13). In order to avoid the ambiguities, we add the superscript ' $n f$ ' for those calculations made for the transformed normal form (25), otherwise it is for the original system (13).

For the transformed normal form (25), we have $\theta_{i, 1}^{n f}=d z_{i, r_{i}}$ and $\theta_{i, j}^{n f}=d z_{i, r_{i}-j+1}$ for $1 \leq i \leq \bar{m}$ and $1 \leq j \leq r_{i}$, which yields $\tau_{i, 1}^{n f}=\frac{\partial}{\partial z_{i, 1}}$ and $\tau_{i, j}^{n f}=\frac{\partial}{\partial z_{i, j}}$ for $1 \leq i \leq \bar{m}$ and $1 \leq j \leq r_{i}$. Then it is clear that $\left[\tau_{i, j}^{n f}, \beta_{k}(y)\right]=0$ for $1 \leq i \leq \bar{m}, 1 \leq j \leq r_{i}-1$ and $1 \leq k \leq \bar{m}$. Thus we proved that if there exists a local diffeomorphism $z=\phi(x)$ on $\bar{\Omega}_{x}$ which transforms (13) into (25), then the third item of Theorem 1 should be satisfied for the transformed normal form (25).

Since $\omega=d \phi$ are the pullbacks of $d z$, due to the fact that $\tau_{i, j}^{n f}=\frac{\partial}{\partial z_{i, j}}$ for $1 \leq i \leq \bar{m}$ and $1 \leq j \leq r_{i}$, by defining

$$
\tau_{i, j}=\phi_{*}^{-1}\left(\tau_{i, j}^{n f}\right), \text { for } 1 \leq i \leq \bar{m}, 1 \leq j \leq r_{i}
$$

\footnotetext{
${ }^{1}$ Do not confuse the differential (Jacobian) of $\phi: \phi_{*}$ where the star is subscript with the pullback: $\phi^{*}$ where the star is superscript.
}

we have

$\left[\tau_{i, j}^{n f}, \beta_{k}(y)\right]=\left[\phi^{*}\left(\tau_{i, j}^{n f}\right), \phi^{*}\left(\beta_{k}(y)\right)\right]=\phi^{*}\left(\left[\tau_{i, j}^{n f}, g_{k}(x)\right]\right)=0$

thus we proved as well $\left[\tau_{i, j}^{n f}, g_{k}(x)\right]=0$ for $1 \leq i \leq \bar{m}, 1 \leq j \leq$ $r_{i}-1$ and $1 \leq k \leq \bar{m}$.

Sufficiency: Consider the multi 1-forms $\omega$ defined in (24), we have $\omega(\tau)=I_{n \times n}$, which implies $\omega\left(\tau_{i, j}\right)$ for $1 \leq i \leq \bar{m}$ and $1 \leq$ $j \leq r_{i}$ are constant. Therefore,

$$
\begin{aligned}
d \omega\left(\tau_{i, j}, \tau_{k, s}\right) & =L_{\tau_{i, j}} \omega\left(\tau_{k, s}\right)-L_{\tau_{k, s}} \omega\left(\tau_{i, j}\right)-\omega\left(\left[\tau_{i, j}, \tau_{k, s}\right]\right) \\
& =-\omega\left(\left[\tau_{i, j}, \tau_{k, s}\right]\right) .
\end{aligned}
$$

Since $\omega$ is an isomorphism, this implies the equivalence between $\left[\tau_{i, j}, \tau_{k, s}\right]=0$ and $d \omega=0$.

According to the theorem of Poincaré, $d \omega=0$ implies that there exists a local diffeomorphism $z=\phi(x)$ such that $\omega=d \phi$. Since the first condition in Theorem 1 is satisfied, we have $\phi_{*}\left(\tau_{i, j}\right)=\frac{\partial}{\partial z_{i, j}}$ for $1 \leq i \leq \bar{m}$ and $1 \leq j \leq r_{i}$. For $1 \leq k \leq \bar{m}$, if $\left[\tau_{i, j}, g_{k}\right]=0$, then

$$
\frac{\partial}{\partial z_{i, j}} \phi_{*}\left(g_{k}\right)=\phi_{*}\left(\left[\tau_{i, j}, g_{k}\right]\right)=0,
$$

which implies $\phi_{*}\left(g_{k}(x)\right)=\beta_{k}(y)$, and finally we proved that (20) can be transformed into (25).

Remark 5. In this paper, no assumption on the uniqueness of solution was impose for the studied system. Concerning observability, system's state can still be observable even multiple/infinity solutions exist. A simple example is E $\dot{x}=0, y=x$ with $E=0$, where $x$ can be any functions, but the output enables us to know all states. In other words, the proposed result in this paper works as well for non-regular singular systems whose solution is not unique. This point will be illustrated in the example in Section 6.

\section{Observer design}

This section focuses on Luenberger-like observer design problem for the transformed normal form in the last section. In a more compact manner, the normal form (25) can be rewritten as follows:

$$
\left\{\begin{array}{l}
\dot{z}=A z+\alpha(y)+\beta(y) \dot{y} \\
y=C z
\end{array}\right.
$$

with $\quad A=\operatorname{diag}\left[A_{1}, \cdots, A_{\bar{m}}\right], \quad \alpha=\left(\alpha_{1}^{T}, \cdots, \alpha_{\bar{m}}^{T}\right)^{T}$, $\beta=\left(\beta_{1}^{T}, \cdots, \beta_{\bar{m}}^{T}\right)^{T}, C=\operatorname{diag}\left[C_{1}^{T}, \cdots, C_{\bar{m}}^{T}\right]^{T} . \quad$ It is clear that the pair $(A, C)$ is observable, which means there exists a constant matrix $G$ such that $(A-G C)$ is Hurwitz.

Assumption 3. It is assumed that $\beta(y)$ in (27) is integrable, i.e.

$$
\exists K(y) \text { such that } \frac{\partial K(y)}{\partial y}=\beta(y)
$$


Remark 6. In general, the integrability of $\beta(y)$ is a strong con- Step 1: Calculate the elementary matrices $T$ and $S$ to transform (5) dition. It is worth noting that this restriction is neither due to the differential geometric method we used, nor due to the singular system we studied. In fact, it is a necessary requirement to design a Luenberger-like observer (like (4)) for any type of systems (linear/nonlinear, regular/singular). This condition is not required in linear case, since $\beta(y)$ in this situation is constant, thus the integrability is always satisfied. It can be seen as well that, for single output nonlinear system, i.e. $y \in \mathbb{R}$, this condition is always true. In summary, this integrability condition appears only for nonlinear systems with multiple outputs.

For the normal form (27), we can then propose the following observer.

Lemma 2. If Assumption 3 is satisfied, then the following $d y$ namics

$$
\left\{\begin{array}{l}
\dot{\xi}=N \xi+L(y) \\
\hat{z}=\xi+K(y)
\end{array}\right.
$$

with

$$
\begin{aligned}
& \frac{\partial K(y)}{\partial y}=\beta(y) \\
& N=A-G C \\
& L(y)=G y+N K(y)+\alpha(y)
\end{aligned}
$$

is an exponential observer for (27).

Proof. Set $e=z-\hat{z}$, then the observation error equals to

$$
\dot{e}=A z+\alpha(y)+\beta(y) \dot{y}-N \hat{z}+N K(y)-L(y)-\frac{\partial K(y)}{\partial y} \dot{y}
$$

Since $\frac{\partial K(y)}{\partial y}=\beta(y)$, then we have

$$
\begin{aligned}
\dot{e}= & (A-G C) e+\alpha(y)+G y-G C \hat{z}+A \hat{z}-N \hat{z} \\
& +N K(y)-L(y)
\end{aligned}
$$

Choose $N=A-G C$, and $L(y)=\alpha(y)+G y+N K(y)$, we obtain

$$
\dot{e}=(A-G C) e
$$

By choosing $G$ such that $(A-G C)$ is Hurwitz, we conclude that (29) is an exponential observer of (27).

Remark 7. It is worth noting that the proposed observer (29) is for the transformed observer normal form (27), i.e., $\lim _{t \rightarrow \infty}\|\hat{z}(t)-z(t)\|=0$. By inverting the local diffeomorphism $z=\phi(x)$ for $x \in \bar{\Omega}_{x} \subseteq \Omega_{x}$, we can then have $\lim _{t \rightarrow \infty} \| \hat{x}(t)-$ $x(t) \|=0$ with $\hat{x}=\phi^{-1}(\hat{z})$. It is clear that the knowledge of $\phi(x)$ and its inverse should be calculated. If the inverse computation of such a diffeomorphism is too complicated, some numerical algorithms, such as Gradient or Newton method Moore (1969), can be applied to solve the following optimization problem: $\hat{x}=\arg \min _{x \in \bar{\Omega}_{x}}\|\hat{z}-\phi(x)\|$.

In summary, by transforming the studied system (5) into (27), if the set of solutions for system (5) is contained in (or equal to) that of (27), then one can construct an observer for (27), and the estimation for (5) can be achieved by inverting the transformation.

Finally, for a given nonlinear singular system (5), the procedure to design a Luenberger-like observer can be summarized as follows:

to semi-explicit form (9);

Step 2: Regularize (9) into a nonlinear regular system (13), if Assumption 1 is satisfied;

Step 3: If Assumption 2 and all conditions of Theorem 1 are satisfied, then deduce a diffeomorphism $z=\phi(x)$ to transform the regularized system (13) into observer normal form (27);

Step 4: Finally, if Assumption 3 is satisfied, then construct the proposed Luenberger-like observer (29) to estimate $z$, which yields $\hat{x}=\phi^{-1}(\hat{z})$, and $\hat{\zeta}=T^{-1} \hat{x}$.

\section{Illustrative example}

Consider a system of the form (5) with

$$
\begin{gathered}
\bar{E}=\left[\begin{array}{cccc}
-4 & 3 & 0 & 0 \\
4 & -1 & 0 & 0 \\
-4 & 2 & 0 & 0 \\
0 & 1 & 0 & 0
\end{array}\right] \\
\bar{f}(\zeta)=\left[\begin{array}{c}
4 \zeta_{1}-4 \zeta_{2}-3 \zeta_{4}+\left(3 \zeta_{2}-\zeta_{3}+2 \zeta_{4}\right)\left(\zeta_{3}+\zeta_{4}\right) \\
4 \zeta_{2}-4 \zeta_{1}+\zeta_{4}+\left(3 \zeta_{3}-\zeta_{2}+2 \zeta_{4}\right)\left(\zeta_{3}+\zeta_{4}\right) \\
4 \zeta_{3}+6 \zeta_{4}+2\left(\zeta_{2}-\zeta_{3}\right)\left(\zeta_{3}+\zeta_{4}\right) \\
-\zeta_{4}+\left(\zeta_{2}+\zeta_{3}+2 \zeta_{4}\right)\left(\zeta_{3}+\zeta_{4}\right)
\end{array}\right]
\end{gathered}
$$

and

$$
\bar{h}(\zeta)=\left[\begin{array}{c}
\zeta_{3}+\zeta_{4}+\underset{2}{2} \zeta_{2} \zeta_{4}+\zeta_{2}^{2}+\zeta_{4}^{2} \\
\zeta_{2}+\zeta_{4}
\end{array}\right]
$$

It is easy to see that the studied system does not satisfy Lipschitz condition since it contains quadratic terms. Therefore, existing results, like those in Shields (1997); Gao and Ho (2006); Darouach and Boutat-Baddas (2008) cannot be applied. By following the presented procedure, the steps to design a nonlinear Luenberger-like observer are summarized as follows.

\section{Step 1:}

Note $\operatorname{rank} \bar{E}=q=2<n=4$, then there exist two elementary matrices $T=\left[\begin{array}{cccc}1 & 0 & 0 & 0 \\ 1 & 1 & 0 & 0 \\ 1 & 1 & 1 & -1 \\ -1 & -1 & 0 & 1\end{array}\right]$ and $S=$ $\frac{1}{4}\left[\begin{array}{cccc}0 & 1 & 0 & 1 \\ 1 & 1 & 1 & 0 \\ 0 & 1 & 1 & -1 \\ 1 & 1 & 0 & -2\end{array}\right]$ such that $E=S \bar{E} T=\left[\begin{array}{cccc}1 & 0 & 0 & 0 \\ 0 & 1 & 0 & 0 \\ 0 & 0 & 0 & 0 \\ 0 & 0 & 0 & 0\end{array}\right]$, thus, by introducing $x=T^{-1} \zeta=\left[\begin{array}{c}\zeta_{1} \\ \zeta_{2}-\zeta_{1} \\ \zeta_{3}+\zeta_{4} \\ \zeta_{2}+\zeta_{4}\end{array}\right]$, this system becomes

$$
\begin{cases}\dot{x}_{1} & =x_{2}+x_{3}^{2} \\ \dot{x}_{2} & =x_{3}+x_{3} x_{4} \\ 0 & =x_{3}-x_{1}+x_{4} \\ \bar{y}_{1} & =x_{3}+x_{4}^{2} \\ \bar{y}_{2} & =x_{4}\end{cases}
$$


Step 2:

It is easy to check that

$$
\operatorname{rank}\left[\begin{array}{cc}
\bar{E} & \frac{\partial \bar{f}(\zeta)}{\partial \zeta} \\
0 & \bar{E} \\
0 & \frac{\partial \bar{h}(\zeta)}{\partial \zeta}
\end{array}\right]=6=4+\operatorname{rank} \bar{E}
$$

thus Assumption 1 is satisfied.

Following the procedure presented in this paper, we can then regularize the above system as follows:

$$
\left\{\begin{aligned}
\dot{x} & =\left[\begin{array}{c}
x_{2}+x_{3}^{2} \\
x_{3}+x_{3} x_{4} \\
x_{2}+x_{3}^{2} \\
0
\end{array}\right]+\left[\begin{array}{c}
0 \\
0 \\
-1 \\
1
\end{array}\right] \dot{y}_{3} \\
y & =\left[\begin{array}{c}
x_{3}-x_{1}+x_{4} \\
x_{3}+x_{4}^{2} \\
x_{4}
\end{array}\right]
\end{aligned}\right.
$$

\section{Step 3:}

By checking Assumption 2, we get $r_{1}=r_{3}=1$ and $r_{2}=2$, such that

$$
\operatorname{rank}\left[\begin{array}{c}
d h_{1} \\
d h_{2} \\
d L_{f} h_{2} \\
d h_{3}
\end{array}\right]=\operatorname{rank}\left[\begin{array}{cccc}
-1 & 0 & 1 & 1 \\
0 & 0 & 1 & 2 x_{4} \\
0 & 1 & 2 x_{3} & 0 \\
0 & 0 & 0 & 1
\end{array}\right]=4
$$

Thus Assumption 2 is true as well.

$$
\text { Then, we can calculate } \theta=\left[\begin{array}{c}
d h_{1} \\
d h_{2} \\
d L_{f} h_{2} \\
d h_{3}
\end{array}\right] \text {, and the correspond- }
$$

ing $\tau$ defined in (23), which enables us to deduce the following diffeomorphism

$$
\left[\begin{array}{c}
z_{1,1} \\
z_{2,1} \\
z_{2,2} \\
z_{3,1}
\end{array}\right]=\left[\begin{array}{c}
x_{3}-x_{1}+x_{4} \\
x_{3}+x_{4}^{2} \\
x_{2}+x_{3}^{2} \\
x_{4}
\end{array}\right]
$$

with which the studied system can be finally transformed into:

$$
\left\{\begin{aligned}
\dot{z}_{1,1}= & 0 \\
\dot{z}_{2,1}= & z_{2,2}-\dot{y}_{3}+2 y_{3} \dot{y}_{3} \\
\dot{z}_{2,2}= & y_{2}-y_{3}^{2}+y_{2} y_{3}-y_{3}^{3} \\
& +2 y_{2} \dot{y}_{2}-4 y_{2} y_{3} \dot{y}_{3}-2 y_{3}^{2} \dot{y}_{2}+4 y_{3}^{2} \dot{y}_{3} \\
\dot{z}_{3,1}= & \dot{y}_{3} \\
y_{1}= & z_{1,1} \\
y_{2}= & z_{2,1} \\
y_{3}= & z_{3,1}
\end{aligned}\right.
$$

thus we have $A=\left[\begin{array}{llll}0 & 0 & 0 & 0 \\ 0 & 0 & 1 & 0 \\ 0 & 0 & 0 & 0 \\ 0 & 0 & 0 & 0\end{array}\right]$ and $C=$

$$
\begin{aligned}
{\left[\begin{array}{llll}
1 & 0 & 0 & 0 \\
0 & 1 & 0 & 0 \\
0 & 0 & 0 & 1
\end{array}\right] \text { and } } \\
\beta(y) \dot{y}=\left[\begin{array}{c}
0 \\
-\dot{y}_{3}+2 y_{3} \dot{y}_{3} \\
2 y_{2} \dot{y}_{2}-4 y_{2} y_{3} \dot{y}_{3}-2 y_{3}^{2} \dot{y}_{2}+4 y_{3}^{2} \dot{y}_{3} \\
\dot{y}_{3}
\end{array}\right]
\end{aligned}
$$

\section{Step 4:}

It can be checked that $\beta(y)$ is integrable since there exists

$$
K(y)=\left[\begin{array}{c}
0 \\
-y_{3}+y_{3}^{2} \\
\left(y_{2}-y_{3}^{2}\right)^{2} \\
y_{3}
\end{array}\right]
$$

such that $\frac{\partial K(y)}{\partial y}=\beta(y)$. Therefore, Assumption 3 is verified.

Finally, we can design a simple Luenberger-like observer of form (29) to exponentially estimate the state. For the simulation, we choose

$$
G=\left[\begin{array}{cccc}
25.6018 & 23.4427 & 259.7463 & 0 \\
6.2637 & 54.3982 & 454.1471 & 0 \\
0 & 0 & 0 & 20
\end{array}\right]^{T}
$$

such that $(A-G C)$ has negative eigenvalues $(-20,-50,-20,-10)$. It can be seen from (30) that the studied example is non-regular singular system since the solution is not unique, which however has no influence to observability, and the studied example is still observable. From (30) it is evident that either $x_{3}$ or $x_{4}$ is free (i.e., it can be any function), and the algebraic equation $0=x_{3}-x_{1}+x_{4}$ governs the other variable once $x_{3}$ or $x_{4}$ is determined. In our simulation, $x_{4}$ was chosen as a free $\cos (t)$ function, and the simulation results of estimation error for $\zeta$ are depicted in Fig. 1.

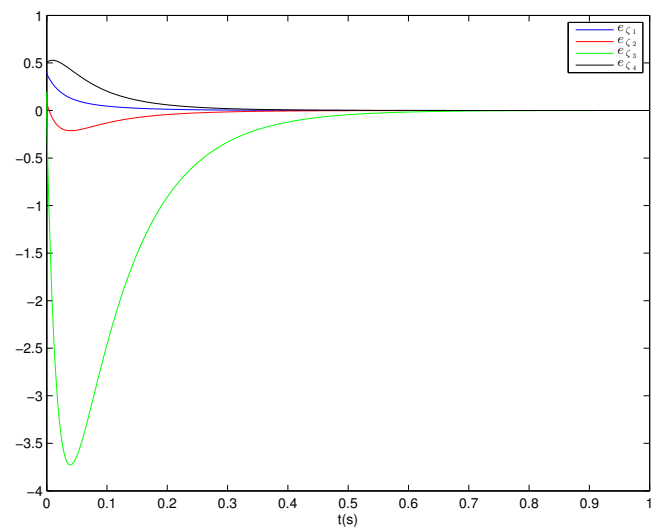

Figure 1: Estimation errors of $\zeta$. 


\section{Conclusion}

This paper generalizes our previous work to treat nonlinear singular systems with multiple outputs. The basic idea is to regularize firstly the nonlinear singular system by injecting the derivative of the outputs into the model. Then, regarding $\dot{y}$ as an input, we can easily reuse the existing result of differential geometric method to transform the regularized system into a simple normal form, which contains as well the derivative of output. Necessary and sufficient conditions are deduced to guarantee such a transformation. Finally, a simple Luenberger-like observer can be designed, provided that the integrability condition is satisfied.

\section{Reference}

Bejarano, F. J., Floquet, T., Perruquetti, W., Zheng, G., 2011. Observability and detectability analysis of singular linear systems with unknown inputs. In: Decision and Control and European Control Conference (CDC-ECC), 2011 50th IEEE Conference on. IEEE, pp. 4005-4010.

Bejarano, F. J., Floquet, T., Perruquetti, W., Zheng, G., 2013. Observability and detectability of singular linear systems with unknown inputs. Automatica 49(3), 793-800.

Bejarano, F. J., Perruquetti, W., Floquet, T., Zheng, G., 2012. State reconstruction of nonlinear differential-algebraic systems with unknown inputs. In: Decision and control (CDC), 2012 IEEE 51st annual conference on. IEEE, pp. 5882-5887.

Bejarano, F. J., Perruquetti, W., Floquet, T., Zheng, G., 2015. Observation of nonlinear differential-algebraic systems with unknown inputs. IEEE Transactions on Automatic Control 60 (7), 1957-1962.

Boutat, D., Zheng, G., Boutat-Baddas, L., Darouach, M., 2012. Observers design for a class of nonlinear singular systems. In: in Proc. IEEE Conf. Decision Control.

Boutayeb, M., Darouach, M., 1995. Observers design for non linear descriptor systems. In: in Proc. IEEE Conf. Decision Control.

Campbell, S., 1980. Singular systems of differential equations I. Pitman.

Campbell, S., 1982. Singular systems of differential equations II. Pitman.

Dai, L., 1989. Singular Control Systems. Vol. 118. Lecture Notes in Control and Information Sciences, Springer-Verlag, New York.

Darouach, M., Boutat-Baddas, L., 2008. Observers for a class of nonlinear singular systems. IEEE Transactions on Automatic Control 53 (11), 26272633.

Darouach, M., Boutayeb, M., 1995. Design of observers for descriptor systems. IEEE Trans. Automatic Control 40(7), 1323-1327.

Gao, Z., Ho, D. W. C., 2004. Proportional multiple-integral observer design for descriptor systems with measurement output disturbances. IEE Proceedings - Control Theory and Applications 151 (3), 279-288.

Gao, Z., Ho, D. W. C., 2006. State/noise estimator for descriptor systems with application to sensor fault diagnosis. IEEE Transactions on Signal Processing 54 (4), 1316-1326.

Hou, M., Muller, P., 1999a. Casual observability of descriptor systems. IEEE Transactions on Automatic Control 44 (1), 158-163.

Hou, M., Muller, P., 1999b. Observer design for descriptor systems. IEEE Transactions on Automatic Control 44 (1), 164-169.

Isidori, A., 1995. Nonlinear Control Systems, 3rd Edition. Springer-Verlag New York, Inc., Secaucus, NJ, USA.

Kaprielian, S., Turi, J., 1992. An observer for a nonlinear descriptor system. in Proc. IEEE Conf. Decision Control.

Koenig, D., 2005. Unknown input proportional multiple-integral oberver design for linear descriptor systems: application to state and fault estimation. IEEE Transactions on Automatic Control 50 (2), 212-217.

Koenig, D., 2006. Observers design for unknown input nonlinear descriptor systems via convex optimization. IEEE Trans. Automatic Control 51(6), 1047-1052.

Koenig, D., Mammar, S., 2002. Design of proportional-integral observer for unknown input descriptor systems. IEEE Transactions on Automatic Control 47 (12), 2057-2062.
Lu, G., Ho, D., 2006. Full-order and reduced-order observer for lipschitz descriptor systems: The unified lmi approach. IEEE.Trans on Circuits and systems-II: Express Briefs 53, 563-567.

Lu, G., Ho, D. W. C., Zheng, Y., Zheng, Y., 2004. Observers for a class of descriptor systems with lipschitz constraint. In: Proceedings of the 2004 American Control Conference.

Moore, R. H., 1969. Analysis of numerical methods (eugene isaacson and herbert bishop keller). SIAM Review 11 (1), 100-101.

Paraskevopoulos, P., Koumboulis, F., August 1992. Observers for singular systems. IEEE Transactions on Automatic Control 37 (8), 1211 - 1215.

Shields, D. N., 1997. Observer design and detection for nonlinear descriptor systems. International Journal of Control 67 (2), 153-168.

Tami, R., Boutat, D., Zheng, G., 2013. Extended output depending normal form. Automatica 49 (7), 2192-2198.

Tami, R., Zheng, G., Boutat, D., Aubry, D., Wang, H., 2016. Partial observer normal form for nonlinear system. Automatica 64, 54-62.

Xiao, X., Gao, W., 1989. Nonlinear observer design by observer error linearization. SIAM Journal on Control and Optimization 27, 199-216.

Yip, E., Sincovec, R., 1981. Solvability, controllability, and observability of continuous descriptor systems. IEEE Transactions on Automatic Control AC-26 (3), 702-707.

Zheng, G., Boutat, D., 2011. Synchronisation of chaotic systems via reduced observers. IET Control Theory \& Applications 5 (2), 308-314.

Zheng, G., Boutat, D., Barbot, J.-P., 2007. Single output-dependent observability normal form. SIAM Journal on Control and Optimization 46 (6), 22422255.

Zheng, G., Boutat, D., Barbot, J.-P., 2009. Multi-output dependent observability normal form. Nonlinear Analysis: Theory, Methods \& Applications 70 (1), 404-418 